\title{
Energy efficiency of a new trifocal intraocular lens
}

\author{
F. Vega \\ fvega@oo.upc.edu
}

\section{F. Alba-Bueno}

\section{S. Millán}

\author{
Department d'Òptica i Optometria, Universitat Politècnica de Catalunya, Violinista Vellsolà 37, 08222 \\ Terrassa, Spain \\ Department d'Òptica i Optometria, Universitat Politècnica de Catalunya, Violinista Vellsolà 37, 08222 \\ Terrassa, Spain
}

Department d'Òptica i Optometria, Universitat Politècnica de Catalunya, Violinista Vellsolà 37, 08222 Terrassa, Spain

The light distribution among the far, intermediate and near foci of a new trifocal intraocular lens (IOL) is experimentally determined, as a function of the pupil size, from image analysis. The concept of focus energy efficiency is introduced because, in addition to the theoretical diffraction efficiency of the focus, it accounts for other factors that are naturally presented in the human eye such as the level of spherical aberration (SA) upon the $10 \mathrm{~L}$, light scattering at the diffractive steps or the depth of focus. The trifocal $10 \mathrm{~L}$ is tested in-vitro in two eye models: the aberration-free ISO model, and a so called modified-ISO one that uses an artificial cornea with positive spherical SA in instead. The SA upon the IOL is measured with a Hartmann-Shack sensor and compared to the values of theoretical eye models. The results show, for large pupils, a notorious reduction of the energy efficiency of the far and near foci of the trifocal IOL due to two facts: the level of SA upon the $10 \mathrm{~L}$ is larger than the value the lens is able to compensate for and there is significant light scattering at the diffractive steps. On the other hand, the energy efficiency of the intermediate focus for small pupils is enhanced by the contribution of the extended depth of focus of the near and far foci. Thus, while IOLs manufacturers tend to provide just the theoretical diffraction efficiency of the foci to show which would be the performance of the lens in terms of light distribution among the foci, our results put into evidence that this is better described by using the energy efficiency of the foci.

[DOI: http://dx.doi.org/10.2971/jeos.2014.14002]

Keywords: Trifocal intraocular lens, diffraction efficiency, cataract, eye model

\section{INTRODUCTION}

Increasing patient demand of spectacle independence after cataract surgery or clear lens extraction has been an extraordinary incentive for the development of multifocal intraocular lenses (MIOLs). [1] Diffracted-based MIOLs have shown better optical performance than either, their counterparts refractive MIOLs or accommodating IOLs. [2,3] Until very recently, diffractive MIOLs available in the market were indeed bifocal lenses that use the base lens curvature and the zero $(m=0)$ and first $(m=1)$ diffraction orders to achieve simultaneous far and near foci respectively. In clinical tests, patients implanted with diffractive MIOLs achieved good far and near visual acuities $[4,5]$ but have a significant reduction of visual acuity at intermediate distance. [6, 7] To deal with this issue, Swanson disclosed a trifocal IOL [8] based on a binary phase profile that used the $m=-1, m=0$ and $m=1$ diffraction orders to obtain the far, intermediate and near foci respectively, each of them having a theoretical diffraction efficiency of $28.8 \%$ while the rest of the light energy (approximately 14\%) was wasted in higher diffraction orders. The Record trifocal IOL, used for the first time in 36 eyes (28 patients) by Vorkresenkaya et al [9] is designed according to these principles. A different approach in the design of trifocal IOLs relies on the combination of two kinoform diffractive profiles with different step heights in the surface of the lens. [10] The first profile provides focus for far (order $m=0$ ) and near (order $m=1$ ) vision, while the second one splits the light into far (order $m=0$ ) and intermediate (order $m=1$ ) foci. Moreover, the order $m=2$ due to this profile coincides with the order $m=1$ of the first one and thus also contributes to the near focus. The Finevision trifocal IOL (PhysIOL, Lige, Belgium) featuring an apodized diffractive profile with add powers of $+1.75 \mathrm{D}$ for intermediate and +3.50D for near, [11] uses such design and the first visual outcomes after implantation of this IOL are now available. $[12,13]$ Finally, a new trifocal diffractive IOL, the AT Lisa tri 839MP (Carl Zeiss, Meditec) has been recently launched. [14] This lens features an aspheric optic for which the light distribution among the foci is intended to be independent from the pupil size. As for any type of multifocal IOL, the trifocal one is based on the simultaneous image forming principle. This means that a focused image (due to one of the foci) will be always overlaid by two out of focus images originated by the others foci. This effect will unavoidably lead to a reduction of the image contrast and likely produce other disturbing optical effects such as glare and/or halos, the latter being intimately related to the way the light is distributed among the foci. [15] For these reasons it is necessary to characterize in-vitro the optical performance of these new trifocal IOLs not only in terms of metrics like the Modulation Transfer Function $[16,17]$ but also determining the energy distribution among the foci and its variation 


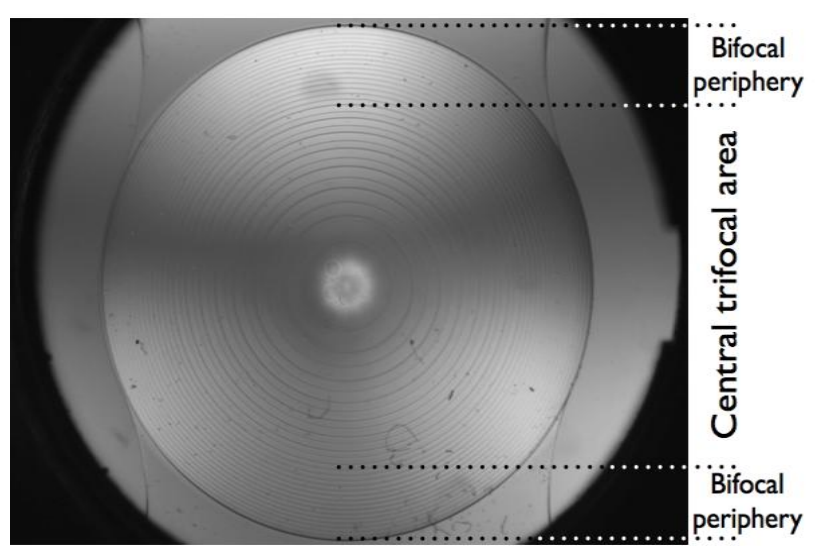

FIG. 1 Optical image of the front surface of the AT LISA tri 839MP IOL. The central trifocal area has the diffractive profile responsible for the formation of the near, intermediate and far foci. The bifocal periphery sends light to the near and far foci.

with the pupil diameter. $[11,18]$ The aim of this paper is to experimentally determine, for the first time to best of our knowledge, the energy efficiency of the far, intermediate and near foci of the AT LISA tri 839MP trifocal IOL as a function of the pupil size. To this end, the IOL is placed in the ISO model eye, [19] which uses an aberration-free achromatic doublet as the artificial cornea. In a second configuration referred from now on to as modified-ISO eye model, the aberration-free cornea is substituted by a double convex lens [20] that provides levels of SA at the IOL plane similar to the ones found in human corneas. [21,22] The model eye with the trifocal IOL forms three images simultaneously (far, intermediate and near) of a pinhole object at infinity. In each of the three image planes, the correctly focused image of the pinhole is surrounded by a blurred halo-shape background that corresponds primarily to the overlaying defocused images due to the other two foci. Image analysis is then used to compute the energy efficiency of each focus through the ratio of the energy correctly focused to the total energy contained in the image.

\section{METHODS}

\subsection{IOL characteristics}

The AT LISA tri 839MP IOL is made of hydrophilic acrylic $(25 \%)$ material with hydrophobic surfaces. The studied lenses have a base optical power for the far focus of 20D with +3.33D and $+1.66 \mathrm{D}$ add powers for the near and the intermediate foci respectively. Multifocality is achieved by means of a diffractive anterior surface of $6 \mathrm{~mm}$ with the trifocal zone having a diameter of $4.34 \mathrm{~mm}$ (Figure 1). The outer region of the lens to the $6 \mathrm{~mm}$ edge sends light to the far and near foci exclusively. In contrast with other trifocal IOLs whose diffractive profile features are currently available, $[9,11]$ to the best of our knowledge no details about the characteristics of the diffractive profile of the AT LISA tri 839MP have been disclosed by the manufacturer. Nevertheless, it is known [14] that this IOL has an aspheric design to produce a negative value of SA $(\mathrm{c}[4,0]$ Zernike coefficient of $-0.18 \mu \mathrm{m}$ for a $6 \mathrm{~mm}$ eye pupil) to compensate in part for the natural positive SA of human cornea.

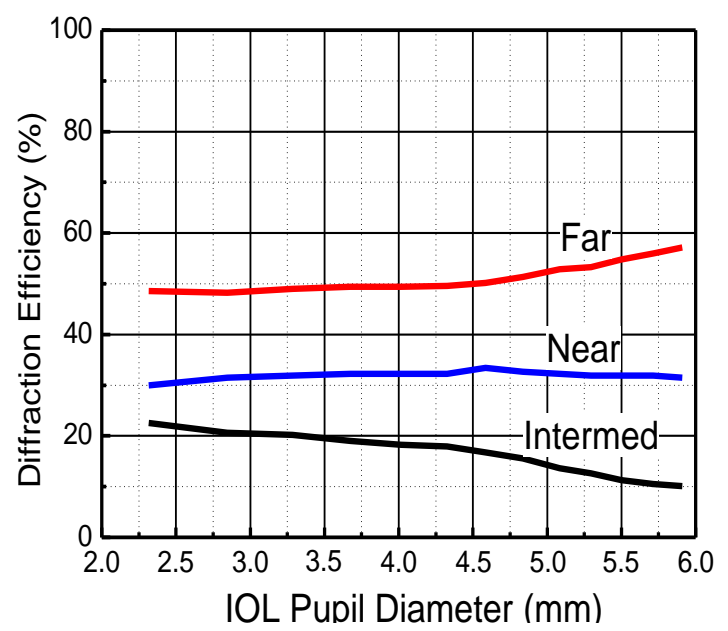

FIG. 2 Diffraction efficiency of the far, intermediate and near foci as provided by the manufacturer in ref [14].

The expected light distribution between the three foci as provided by the manufacturer is shown in Figure 2. It is worth emphasizing that this distribution of energy is indeed the theoretical diffraction efficiency $[15,23]$ of the far, intermediate and near foci of the IOL. Since the amount of the energy transmitted by the trifocal IOL and sent to each of the foci is proportional to this diffraction efficiency, it is generally assumed that the theoretical light distribution showed in Figure 2 will correspond to the energy correctly focused onto the respective image planes. However, in addition to the diffraction efficiency of each of the foci, there are other factors that determine how much of the diffracted energy really ends up correctly focused in the image planes. Thus, it has been pointed out the importance of the shape factor and aspheric design [24,25] of the base lens of the IOL to properly tackle with the converging and aberrated wavefront $[26,27]$ that impinges upon the IOL in order to obtain a high quality retinal image. Additional issues such as the presence of higher diffraction orders (i.e., others than the ones that create the three foci), [23] scattering in the diffractive steps $[28,29]$ or the dependence of the depth of focus on the pupil size, also make that a significant fraction of the diffracted energy, which otherwise would be sent to a particular focus, gets out of focus at the image plane. This extra out of focus energy increases the background noise of the image and may reduce the efficiency of the foci [18] below the values that are predicted just taking into consideration their theoretical diffraction efficiency. Then, the performance of the foci is better characterized by measuring the energy correctly focused in each image plane, i.e., in terms of their energy efficiency since it accounts not only for the diffraction efficiency of the foci but for all the rest of factors mentioned above.

\subsection{Experimental setup}

The energy efficiency of the far, intermediate and near foci can be obtained through image analysis using the setup sketched in Figure 3 that has been described in detail elsewhere. [30]

The light source is a green Led with their emission centered at $521 \mathrm{~nm}$ and with a FWHM spectral bandwidth of $\pm 15 \mathrm{~nm}$. The maximum output power is $12 \mathrm{~mW}$. The collimated beam illuminates the model eye (artificial cornea plus wet cell) where 


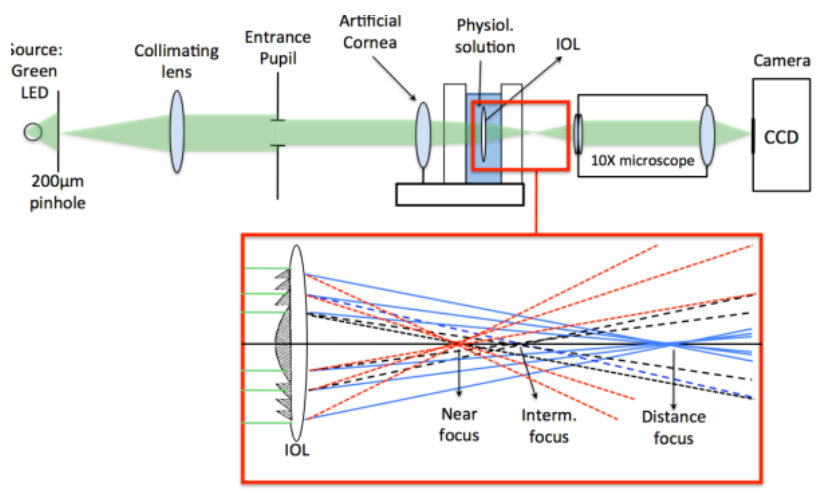

FIG. 3 Setup used to obtain in-vitro the far, intermediate and near images formed by the trifocal IOL. The artificial cornea is either an aberration-free achromatic doublet (ISO model eye) or a double convex lens (modified-ISO model eye). The inset shows how the trifocal IOL works.

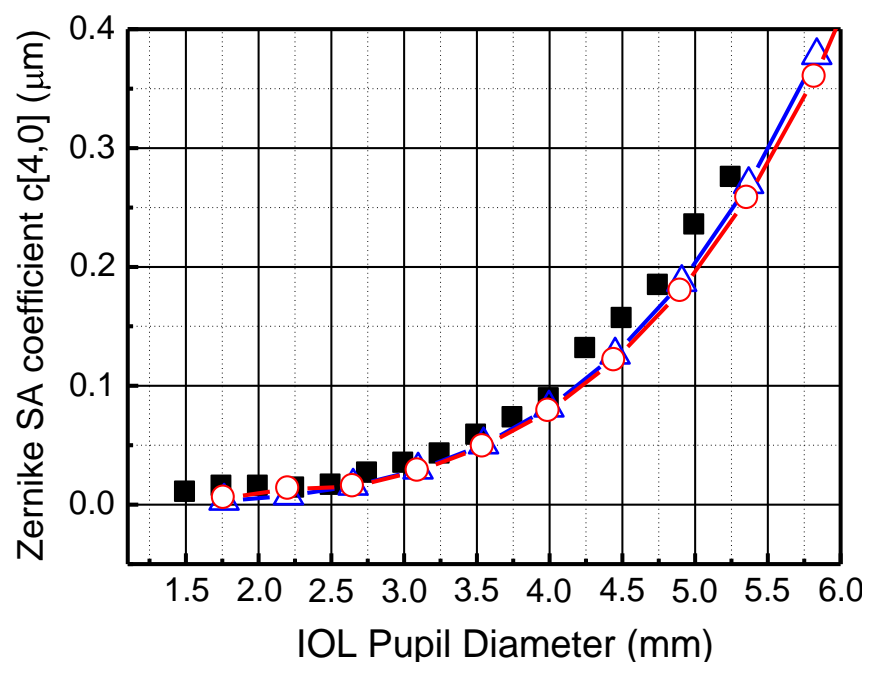

FIG. 4 Values of the Zernike $c[4,0]$ SA coefficient as a function of the IOL-pupil diameter measured in the modified-ISO model eye ( $\boldsymbol{\square})$, and obtained from simulation in the Holladay $(-\bigcirc-\bigcirc-)$ and Liou-Brennan $(-\triangle-\triangle-$ ) eye models.

the trifocal IOL is inserted. The features of both, the ISO and the modified-ISO eye models have been reported elsewhere. $[18,19]$ The pinhole object is imaged in three planes separated along the optical axis (these images will be hereafter referred to as far, intermediate and near, respectively). An infinite corrected microscope mounted in a translation holder is used to select the proper image and magnify it onto an 8-bit CCD camera. The microscope objective is a $10 \times$ Olympus Plan Achromat, designed for high-quality imaging applications due to its diffraction limited performance across the entire visible spectrum. The efficiency of the CCD camera is practically flat along the spectral bandwidth of the green Led source. A variable aperture diaphragm, placed in front of the artificial cornea, is used as the entrance pupil (EP) to control the size of the beam on the artificial cornea and thus the level of SA introduced by the modified-ISO eye model (without the IOL). Additionally, when the trifocal IOL is inserted, the EP diameter also determines the beam size on the lens (referred hereafter to as the IOL-pupil) as described in detail in Reference [30]. In the case of the modified-ISO model, we measured with a HartmannShack sensor (HASO-76, Imagine Optics), the SA of the wavefront that impinges on the trifocal IOL. The results, expressed in terms of the Zernike c[4,0] coefficient are plotted in Figure 4.

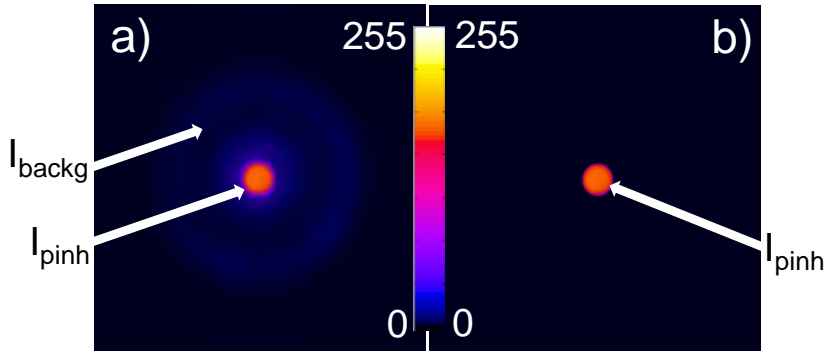

FIG. 5 a) Near image of a pinhole object at infinity experimentally obtained with the trifocal IOL in the modified-ISO model eye. The arrows point out to the regions named pinhole (pinh) and background (backg) respectively. Pseudocolor is used for a better visualization of the contrast in the background. b) same as above after removing the background.

We have also used commercial optical design software (Zemax Development Corporation, San Diego) to obtain the SA in two physiological eye models, namely the Liou-Brennan and the Holladay models. [31] The results, also plotted in Figure 4, show a good agreement with the experimental values, thus confirming that the modified-ISO model provides levels of SA similar to the values found in the aforementioned physiological eye models and measured in human corneas. [21,22]

\subsection{Foci energy efficiency from image analysis}

The method used to obtain the energy efficiency of each of the foci from image analysis has been reported in detail elsewhere [18]. To illustrate the procedure we show in Figure 5 an example of a recorded image corresponding to the near focus of the trifocal IOL.

As previously stated, the image consists of the focused image of the pinhole object (labeled $I_{\text {pinh }}$ in Figure 5(a)) surrounded by a blurred halo-shape background (labeled $I_{b a c k g}$ in Figure 5(a)). The energy of the image just in the focused pinhole region $\left(I_{\text {pinh }}\right)$, and the energy of the total image that comprises the pinhole plus the background region $\left(I_{\text {total }}=I_{\text {pinh }}+I_{\text {backg }}\right)$, are obtained as a function of the IOL-pupil, by integration of the pixel grey level in the corresponding regions:

$$
I_{R}=\sum_{n \text { pixel }}^{n \in R} g(n),
$$

where $R$ stands for either the pinhole region or the total image ( $R=$ pinh,total), $n$ is a pixel contained in the $R$ region, and $g(n)$ is the pixel grey level, which is proportional to the energy impinging on that pixel. It was checked that the CCD sensor response was linear in the dynamic range of interest. Since the images are blurred because of the background, it is necessary to precisely determine the borders of the region that corresponds to the focused pinhole. An edge detection algorithm was used to unambiguously define a region of interest (ROI) that matches with the focused pinhole and remove all the background contribution outside this ROI (Figure 5(b)). Then, the energy correctly focused in the image plane $\left(\left(I_{\text {pinh }}\right)_{n e a r}\right.$ in this case) is calculated by applying Eq. (1) to the filtered image. Finally, the energy efficiency of the focus is obtained as 


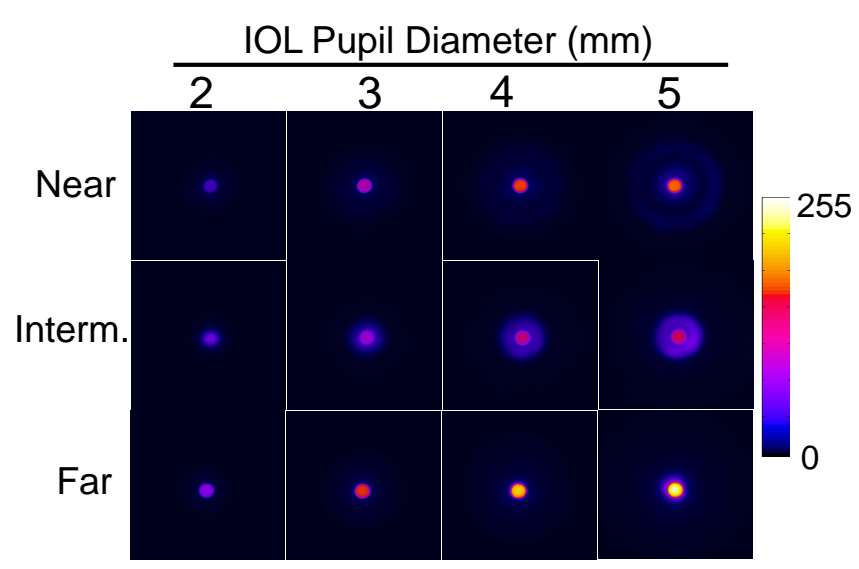

FIG. 6 Near, intermediate and far images of a pinhole object at infinity experimentally obtained with $10 \mathrm{~L}$-pupil diameters ranging from $2 \mathrm{~mm}$ up to $5 \mathrm{~mm}$. The trifocal $10 \mathrm{~L}$ was placed in the modified-ISO model eye. Pseudocolor is used for a better visualization of the images.

the ratio:

$$
(\eta)_{\text {near }}=\frac{\left(I_{\text {pinh }}\right)_{\text {near }}}{\left(I_{\text {total }}\right)_{\text {near }}}
$$

An identical procedure is followed with the images recorded in the intermediate and far image planes to obtain $(\eta)_{\text {intermed }}$ and $(\eta)_{\text {far }}$ respectively.

\section{RESULTS}

Figure 6 shows the experimental images of the pinhole object obtained in the near, intermediate and far image planes for IOL-pupils ranging from 2 to $5 \mathrm{~mm}$. The trifocal IOL was placed in the modified-ISO eye model and all the images were acquired with the same integration time by the CCD camera and thus, their energy content and features can be directly compared. Quite similar images were obtained with the IOL in the ISO eye model.

Figure 6 shows that for each of the foci, the larger the IOLpupil the more the energy correctly focused on the image of the pinhole. However, this is also accompanied by an increasingly notorious background that negatively affects the energy efficiency as will be shown below. By comparing the images it is evident that for a given IOL-pupil, the highest energy in the region of the pinhole occurs in the far focus and the lowest in the intermediate focus. Moreover, there is a very different evolution of the halo-shape background in each of the foci. The halo with the largest size is found in the near focus, especially for the largest IOL-pupils where a double ring feature can be clearly observed from the images. As for the far focus, the halo is both less extended and less notorious. Finally, the images corresponding to the intermediate focus show a clear halo surrounding the image of the pinhole for all IOL-pupil sizes. The total energy of the images $\left(I_{\text {total }}\right)_{i}$, and the energy correctly focused $\left(I_{\text {pinh }}\right)_{i}$, obtained in the three image planes ( $i=$ near, intermed, far) with the trifocal IOL placed in the modified-ISO eye model are plotted, as a function of the IOLpupil diameter, in Figure 7.

To make the comparison of results easier, $\left(I_{\text {total }}\right)_{i}$ and $\left((I)_{\text {pinh }}\right)_{i}$ are normalized for each focus to the maximum

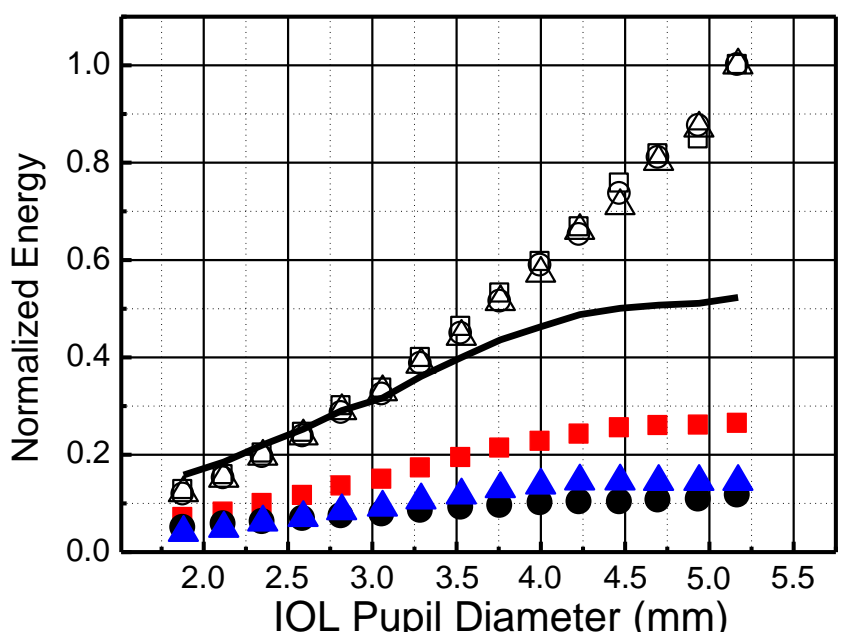

FIG. 7 Total energy of the image $I_{\text {total }}$ (open symbols), and energy contained in the pinhole region $I_{\text {pinh }}$ (solid symbols) measured in the $(\Delta, \boldsymbol{\Delta})$ near, $(0, \boldsymbol{0})$ intermediate and $(\square, \square)$ far image planes of the trifocal IOL as a function of the pupil diameter. Black solid line: sum of the correctly focused energy of the three foci $\left(I_{\text {pinh }}\right)_{\text {near }}+\left(I_{\text {pinh }}\right)_{\text {intermed }}+\left(I_{\text {pinh }}\right)_{\text {far }}$ (see text for details).

value of $\left(I_{\text {total }}\right)_{i}$ obtained with the largest IOL-pupil diameter. In each of the three image planes, the energy contained in the whole image $\left(\left(I_{\text {total }}\right)_{i}\right)$ is the same within the experimental uncertainty. This fact further confirms that there is no loss of energy when recording the images formed by the three foci and guarantees that the normalization of the results by the maximum of $\left(I_{\text {total }}\right)_{i}$ is correct. As for the energy correctly focused $\left(I_{\text {pinh }}\right)$, the far focus achieves the highest one for all pupil sizes. Near and intermediate foci have similar energy for small IOL-pupils while for larger ones the near focus is slightly more intense. Interestingly, for IOL-pupils up to $3 \mathrm{~mm}$ the sum of the correctly focused energy of the three foci $\left(\left(I_{\text {pinh }}\right)_{\text {near }}+\left(I_{\text {pinh }}\right)_{\text {intermed }}+\left(I_{\text {pinh }}\right)_{\text {far }}\right.$-see Figure 7 continuous line-), agrees pretty well with the value of the total energy contained in the images $I_{\text {total }}$, but for larger IOL-pupils this sum is clearly lower than $I_{\text {total }}$. Moreover, Figure 7 also shows that $\left(I_{\text {pinh }}\right)_{\text {near }},\left(I_{\text {pinh }}\right)_{\text {intermed }}$ and $\left(I_{\text {pinh }}\right)_{\text {far }}$ increase as a function of the IOL-pupil but their increase is slow and with a smaller slope than the total energy of the images $\left(I_{\text {total }}\right)$. This fact implies a reduction of the efficiency of the foci for larger pupil diameters as it is shown below. The experimental image energy efficiencies for each focus, obtained using Eq. (2), are plotted in Figure 8 when the trifocal IOL is placed in either the modified-ISO eye model (Figure 8(a)) or the ISO one (Figure $8(\mathrm{~b})$ ). For the sake of comparison the diffraction efficiencies provided by the manufacturer are also included.

In the former eye model and for IOL-pupils up to $3.25 \mathrm{~mm}$, i.e. in conditions that correspond to reduced levels of SA upon the IOL and few diffractive zones of the lens contributing to the diffraction process (see Figure 1), the energy efficiency of the far and near foci are around 50\% and 30\% respectively, and these values are still close to the theoretical diffraction efficiency values. On the other hand, the intermediate focus shows energy efficiency larger than the provided diffraction efficiency.

As for IOL-pupils larger than $3.25 \mathrm{~mm}$, and consequently 


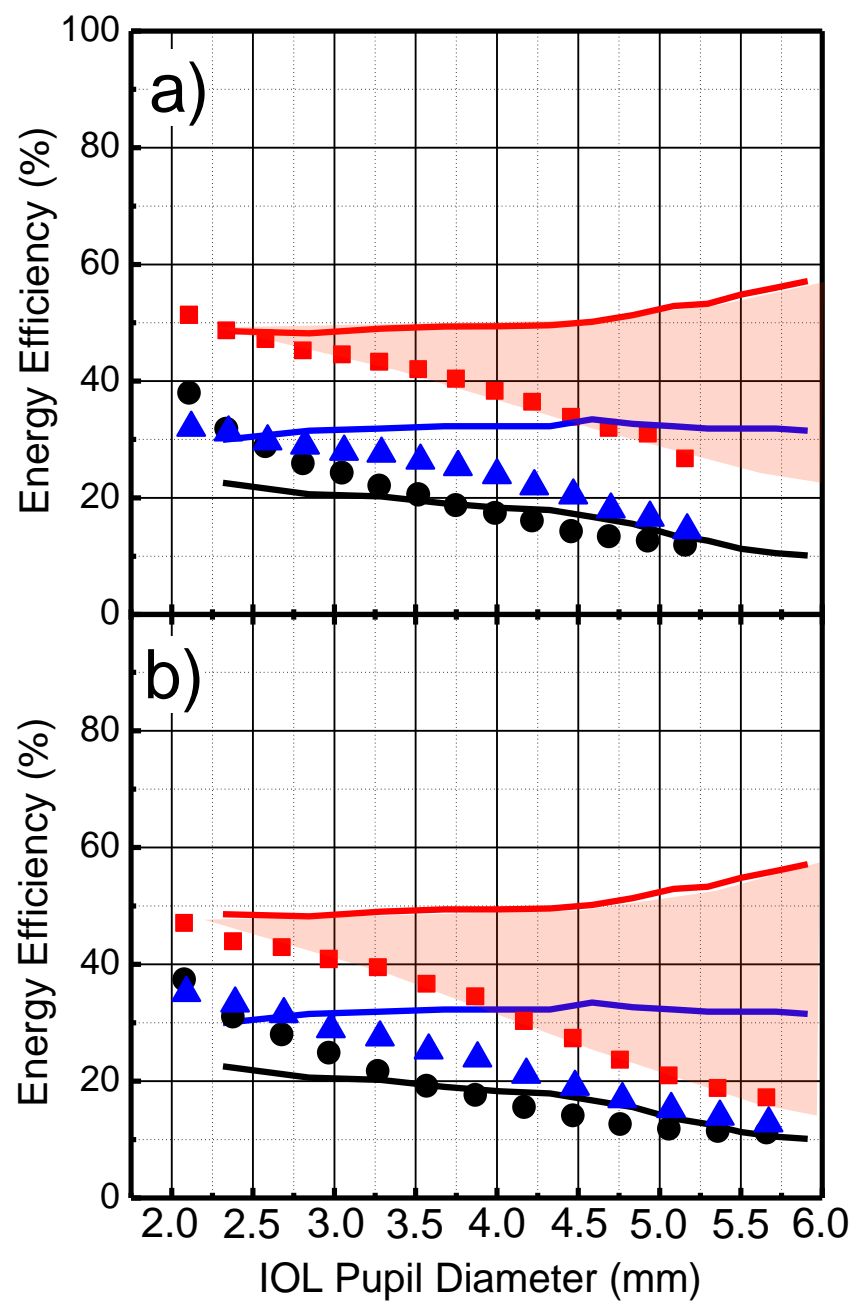

FIG. 8 Energy efficiency obtained according to Equation (2) for the ( $\boldsymbol{\Delta}$ ) near,

( ) intermediate and ( $\square$ ) far foci of the trifocal IOL as a function of the IOL-pupil diameter, obtained in (a) the Modified-ISO model eye and (b) the ISO model. Provided values of the near, intermediate and far diffraction efficiencies, previously showed in Figure 2, are included as blue, black and red solid lines respectively. The shadow areas are to emphasize the differences between measured and provided results for the far focus.

higher levels of SA upon the trifocal IOL and more diffractive zones involved in the process, there is a significant reduction in the experimental efficiencies of the far and near foci in comparison to the values purely based on the theoretical diffraction efficiency of the foci. This reduction is even larger (see Figure $8(\mathrm{~b})$ ) when the trifocal IOL is tested in the ISO-model eye where the artificial cornea is an aberration-free lens. In the case of the intermediate focus, its experimental energy efficiency as a function of the IOL-pupil is very similar in both eye models with values that are in good agreement with the theoretical ones provided by the manufacturer.

\section{DISCUSSION}

The images obtained in each of the three image planes (Figure 6) evidence that there is a non-negligible fraction of the energy that is out of focus, especially for larger IOLpupils, thus contributing to a background noise that reduces the image contrast. Our experimental study of the trifocal IOL performance has the advantage of including, in addition to the diffraction efficiency of each of the orders (or foci), others factors that do affect their energy efficiency. These factors are basically the level of SA upon the IOL and its compensation to some extend because of the aspheric design of the trifocal IOL, the loss of energy due to light scattering at the diffraction steps, and the extended depth of focus for small pupils. Let us start discussing the case of the near and far foci. As stated above, in the far image plane the total energy of the image is:

$$
\left(I_{\text {total }}\right)_{f a r}=\left(I_{\text {pinh }}\right)_{f a r}+\left(I_{\text {backg }}\right)_{f a r}
$$

Interestingly, our results show (Figure 6) that for small IOLpupils (diameters up to $3.0 \mathrm{~mm}$ ):

$$
\left(I_{\text {total }}\right)_{\text {far }} \approx\left(I_{\text {pinh }}\right)_{\text {far }}+\left(I_{\text {pinh }}\right)_{\text {intermed }}+\left(I_{\text {pinh }}\right)_{\text {near }}
$$

and from Eqs. (3) and (4) one infers that:

$$
\left(I_{\text {backg }}\right)_{\text {far }} \approx\left(I_{\text {pinh }}\right)_{\text {intermed }}+\left(I_{\text {pinh }}\right)_{\text {near }},
$$

i.e., the background noise in the far image plane is basically due to the contribution of the other two orders, which are out of focus in that plane but previously were correctly focused in their respective image planes (intermediate and near). A similar reasoning applies to the near focus. Interestingly, this happens for small IOL-pupils, when the aspheric profile of the trifocal IOL must be effective to compensate for the reduced level of SA of the wavefront impinging upon the trifocal IOL (see Figure 4). Moreover, for such a small pupils, the number of diffractive steps that are taking part in the process of diverting light to the foci is small and so it is the light scattering associated with the steps of the diffraction profile. [29] Thus, the experimental near and far energy efficiencies agree reasonably well with the values of the diffraction efficiencies provided by the manufacturer (see Figure 8(a)). For IOL-pupils larger than $3.0 \mathrm{~mm}$, our results show a remarkable reduction of the near and far energy efficiencies (Figure 8). To explain this fact let us insist on the reasoning outlined above. Now, according to Figure 6:

$$
\left(I_{\text {total }}\right)_{\text {far }} \geq\left(I_{\text {pinh }}\right)_{\text {far }}+\left(I_{\text {pinh }}\right)_{\text {intermed }}+\left(I_{\text {pinh }}\right)_{\text {near }},
$$

and from Eqs. (3) and (6):

$$
\left(I_{\text {backg }}\right)_{\text {far }} \geq\left(I_{\text {pinh }}\right)_{\text {intermed }}+\left(I_{\text {pinh }}\right)_{\text {near }},
$$

i.e., in the far image plane the background energy has an additional contribution that does not come from the out of focus near and intermediate orders and as a consequence, there is a significant reduction of the energy efficiency. A similar reasoning applies for the near focus. To explain these results, one must firstly consider the increasing values of the SA of the wavefront upon the trifocal IOL (Figure 4) and secondly, the larger number of diffractive steps taking part in the process (and the associated light scattering). Both effects negatively affect the capacity of the lens to correctly focus the energy diffracted on each order. Additionally, the aspheric design of the AT Lisa tri 839MP IOL is intended to compensate for a maximum positive SA of $0.18 \mathrm{~m}$, compensation that according to Figure 4 can be achieved for IOL-pupils up to $4.75 \mathrm{~mm}$ approximately. Actually, the experimental energy efficiency of the near and far foci of the trifocal IOL is better 
when it is placed in the modified-ISO model (positive SA aberrated cornea) than in the ISO (aberration free cornea) model (the shadow area in Figure 8(a) is smaller than in Figure 8(b)). This fact evidences that the aspheric design of the trifocal IOL is indeed able to compensate (at least partially) for the positive SA of the impinging wavefront and, as a consequence, makes it possible to obtain a better focusing of the energy in the far and near images in the modified-ISO model than in the ISO eye model. [32] However, even for IOL-pupils between 3.0 and $4.75 \mathrm{~mm}$, i.e. when the mechanism of compensation of the SA by the trifocal IOL must be totally effective in the modified-ISO model, the performance of the far and near foci are clearly worse than the diffraction efficiency provided by the manufacturer (Figure 8(a)). In other words, a significant fraction of the additional energy, ideally available for the far and near images when the IOL-pupil increases, does not end up correctly focused in the far and near pinhole images but spoiled in the background of their respective image planes. There must be then another factor (or factors) contributing to the reduction of the efficiency of the far and near foci for IOL-pupil sizes larger than $3.0 \mathrm{~mm}$. If the diffractive profile in the central trifocal area of the AT Lisa tri 839MP IOL (see Figure 1) were based on a pair of kinoforms profiles as in the case of the FineVision IOL, [11] this would imply that any diffractive zone of the lens would consist of at least two diffractive steps (instead of just one as in bifocal diffractive IOLs). Moreover, the provided light distribution (Figure 2) and the claim of the manufacturer of a pupil independent design, [14] lead us to infer that the diffractive profile of the AT Lisa tri 839MP IOL is not apodized. Thus, a significant fraction of the energy would scatter at the large number of non apodized steps and would end up as background noise in the image planes. Finally, additional (although presumably small) contributions to the background may also come from higher diffraction orders. [23] The performance of the intermediate focus deserves further explanation. We have found that for IOL-pupils up to $3.0 \mathrm{~mm}$ approximately, the experimental energy efficiency of this focus is, somehow surprising, larger than the provided values of diffraction efficiency (Figure 8), the smaller the pupil the larger the difference. To explain this fact we refer to Figure 9. The intermediate focus is between the far and near foci and for such small IOL-pupils ( 2.0 to $3.0 \mathrm{~mm}$, where the effects of the SA and light scattering have proved to play a minor role), it is well known that the depth of focus increases. [33] Thus, the smaller the pupil the more extended the depth of focus of the far and near foci and, as a consequence, the smaller the fraction of the energies $\left(I_{\text {pinh }}\right)_{\text {near }}$ and $\left(I_{\text {pinh }}\right)_{\text {far }}$ that are out of focus at the intermediate plane (see Figures 9(b) and 9(c)). This fact becomes more evident when the focal segment corresponding to the depth of focus of both the near and far foci reach one another or even overlap at the intermediate focus plane (Figure 9(c)). The result is a reduction of the energy of the background in the intermediate image plane and, consequently, there is an increase in the relative energy of this focus as experimentally observed. Obviously, this effect related to the extended depths of focus of the far and near foci is not accounted in the provided results that are only based on the diffraction efficiency of the intermediate focus. For IOL-pupils larger than $3.0 \mathrm{~mm}$ the measured energy efficiency of the intermediate focus in both eye models shows a reduction that is in good agreement with the values

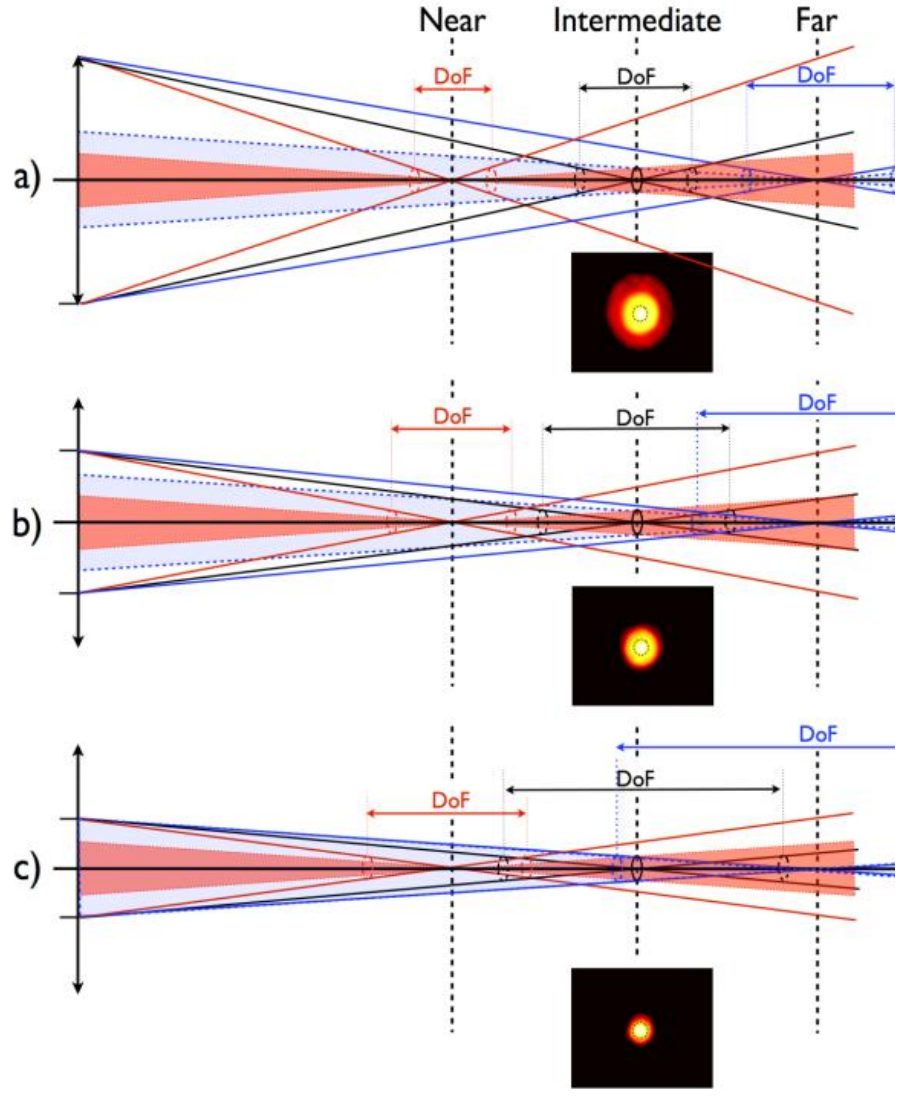

FIG. 9 Sketch of the increasing of the depth of focus as the pupil size is reduced (from $9($ a) to $9(\mathrm{c})$ ). The images at the intermediate plane are shown in a logarithmic scale for the sake of visualization.

of diffraction efficiency. This is not unexpected taking into account that the maximum aperture involved in the formation of the intermediate image is relatively small and so it is the maximum level of SA (Figure 4) that the trifocal IOL has to compensate for. Moreover, once that this zone of the trifocal IOL is fully illuminated, there is no way to diffract more energy to the intermediate correctly focused image $\left(I_{\text {pinh }}\right)_{\text {intermed }}$ and the increase of the total energy of the image $\left(I_{\text {total }}\right)_{\text {intermed }}$ that happens for larger IOL-pupils (Figure 7) leads to the observed reduction of its efficiency.

Finally, it is worth mentioning that the assessment of the IOL energy efficiency with white light illumination would be of enormous interest since this is the type of light that patients implanted with these IOLs use during their daily activities. However, such a broadband source would introduce important challenges in the correct realization and interpretation of the experimental results. First, to avoid wavelengthdependent measurement artifacts, it would be necessary both, a white light source with a uniform spectral emission intensity in the visible range, and a CCD camera with a flat sensitivity along this spectral interval. Secondly, there is an important issue related to the strong dependence on wavelength of two key features of the IOL, namely, the efficiency of the diffraction orders and their associated add powers. [18, 23] This would introduce significant lateral color aberration causing additional blur on all the foci. Consequently, a larger disagreement would result for the experimental energy efficiency of the foci of the trifocal IOL with respect to idealized diffraction efficiency calculations. 


\section{CONCLUSIONS}

In-vitro testing of a new trifocal IOL, the AT LISA tri 839MP, has shown that the light distribution between the far and near foci of the IOL depends, in addition to their theoretical diffraction efficiency, on factors such as the level of SA upon the IOL and light scattering at the diffractive steps. In particular, for IOL-pupils larger than $3.0 \mathrm{~mm}$ the performance of these foci in terms of energy efficiency departs significantly from the values of diffraction efficiency provided by the manufacturer. On the other hand, the intermediate focus efficiency for small IOL-pupils shows a noticeable improvement with respect to the provided values due to the contribution of the extended depths of focus of the near and far foci.

\section{ACKNOWLEDGEMENT}

This study was supported by project DPI2009-08879 from the Spanish Ministerio de Ciencia e Innovación y Fondos FEDER.

\section{References}

[1] N. de Vries, and R. Nuijts, "Multifocal intraocular lenses in cataract surgery: literature review of benefits and side effects," J. Cataract Refr. Surg. 39, 268-278 (2013).

[2] J. M. Artigas, J. L. Menezo, C. Peris, A. Felipe, and M. Díaz-Llopis, "Image quality with multifocal intraocular lenses and the effect of pupil size: Comparison of refractive and hybrid refractivediffractive designs," J Cataract Refr. Surg. 33, 2111-2117 (2007).

[3] W. A. Maxwell, S. S. Lane, and F. Zhou, "Performance of presbyopia correcting intraocular lenses in distance optical bench tests," J. Cataract Refr. Surg. 35, 166-171 (2009).

[4] J. L. Alió, B. Elkady, D. Ortiz, and G. Bernabeu, "Clinical outcomes and intraocular optical quality of a diffractive multifocal intraocular lens with asymmetrical light distribution," J. Cataract Refr. Surg. 34, 942-948 (2008).

[5] J. F. Blaylock, Z. Si, and C. Vickers, "Visual and refractive status at different focal distances after implantation of the ReSTOR multifocal intraocular lens," J. Cataract Refr. Surg. 32, 1464-1473 (2006).

[6] J. C. Alfonso, L. Fernández-Vega, BM. Begoña, and R. Montés-Micó, "Prospective visual evaluation of apodized diffractive intraocular lenses," J Cataract. Refr. Surg. 33, 1235-1243 (2007).

[7] K. Petermeier, and P. Szurman, "Subjective and objective outcome following implantation of the apodized diffractive AcrySof ReSTOR," Ophthalmology 104, 406-408 (2007).

[8] G. J. Swanson, "Diffractive trifocal intraocular lens design," U.S. Patent 5,344,447 (1994).

[9] A. Vorkresenkaya, N. Pozdeyeva, N. Pashtaev, Y. Batkov, V. Treushnicov, and V. Cherednik, "Initial results of trifocal diffractive IOL implantation," Graef. Arch. Clin. Exp. 248, 1299-1306 (2010).

[10] A. L. Cohen, "Diffraction IOL with micromodulation," U.S. Patent 0224138 (2012).

[11] D. Gatine, C. Pagnoulle, Y. Houbrechts, and L. Gobin, “Design and qualification of a diffractive trifocal optical profile for intraocular lenses," J. Cataract Refr. Surg. 37, 2060-2067 (2011).
[12] B. Cochener, J. Vryghem, P. Rozot, G. Lesieur, S. Heireman, J. A. Blanckaert, E. Van Acker, and S. Chekiere, "Visual and refractive outcomes after implantation of a fully diffractive trifocal lens," Clin. Exp. Ophthalmol. 6, 1421-1427 (2012).

[13] A. L. Sheppard, S. Shah, U. Bhatt, G. Bhogal, and J. S. Wolffsohn, "Visual outcomes and subjective experience after bilateral implantation of a new diffractive trifocal intraocular lens," J. Cataract Refr. Surg.39, 343-349 (2013).

[14] J. F. Montin," Achieving spectacle independence with the ATLISA tri 839MP," Cataract at Refractive Surgery Today Europe, March supplement, Vol. 3, 16-19 (2012).

[15] V. Portney, "Light distribution in diffractive multifocal optics and its optimization," J. Cataract Refr. Surg. 37, 2053-2059 (2011).

[16] D. Gatinel, and Y. Houbrechts, "Comparison of bifocal and trifocal diffractive and refractive intraocular lenses using an optical bench," J. Cataract Refr. Surg. 39, 1093-1099 (2013)

[17] J. Ruiz-Alcozer, D. Madrid-Costa, S. García-Lázaro, T. Ferrer-Blasco, and R. Montés-Micó, "Optical performance of two new trifocal intraocular lenses: trough-focus MTF and influence of pupil size," Clin. Exp. Ophthalmol. doi:10.111/ceo.12181.

[18] F. Vega, F. Alba-Bueno, and MS. Millán, "Energy distribution between distance and near images in apodized diffractive multifocal intraocular lenses," Invest. Ophth. Vis. Sci. 52, 5695-5710 (2011).

[19] International Organization for Standardization (ISO), Ophthalmic Implants, Intraocular lenses Part 2: Optical Properties and Test Methods (ISO 11979-2, 1999).

[20] F. Vega, MS. Millán, and B. Wells, "Spherical lens versus aspheric artificial cornea for intraocular lens testing," Opt. Lett. 35, 1539-1541 (2010).

[21] A. Guirao, M. Redondo, and P. Artal, "Optical aberrations of the human cornea as a function of age," J. Opt. Soc. Am. A. 17, 1697-1702 (2000).

[22] L. Wang, E. Dai, DD. Koch, and A. Nathoo, "Optical aberrations of the human anterior cornea," J. Cataract Refr. Surg. 29, 1514-1521 (2003).

[23] F. Castignoles, M. Flury, and T. Lepine, "Comparison of the efficiency, MTF and chromatic properties of four diffractive bifocal intraocular lens designs," Opt. Express 18, 5245-5256 (2010).

[24] J. Tabernero, P. Piers, and P. Artal, "Intraocular lens to correct corneal coma," Opt. Lett. 32, 406-408 (2007).

[25] T. Kohnen, OK. Klaproth, and J. Bühren, "Effect of intraocular lens asphericity on quality of vision after cataract removal: An intraindividual comparison," Ophthalmology 116, 1697-1706 (2009).

[26] S. Pieh, W. Fiala, A. Malz, and W. Stork, "In vitro strehl ratios with spherical, aberration-free, average, and customized spherical aberration-correcting intraocular lenses," Invest. 0phth. Vis. Sci. 50, 1264-1270 (2009).

[27] S. Marcos , P. Rosales, L. Llorente, and I. Jimenez-Alfaro, "Change in corneal aberrations after cataract surgery with two types of aspheric intraocular lenses," J. Cataract. Refr. Surg. 33, 217-226 (2007).

[28] N. E. de Vries, L. Franssen, C. A. B. Webers, N. G. Tahzib, Y. Y. Y. Cheng, F. Hendrikse, K. F. Tjia, et al., "Intraocular straylight after implantation of the multifocal AcrySof ReSTOR SA60D3 diffractive intraocular lens," J. Cataract. Refr. Surg. 34, 957-962 (2008).

[29] M. M. Meyers, and R. E. Albrecht, "Technique to eliminate scattered light in diffractive optical elements," U.S Patent 5,801,889. (1998). 
[30] F. Alba-Bueno, F. Vega, and M. S. Millán, "Design of a test bench for intraocular lens optical characterization," J. Phys. Conf. Ser. 274, 012105-012112 (2011).

[31] S. Norrby, P. Piers, C. Campbell, and M. Van der Mooren, "Model eyes for evaluation of intraocular lenses," Appl. Optics 46, 6595-6605 (2007).
[32] S. Norrby, "Iso eye model not longer valid for assessing aspherical lenses," J. Cataract Refr. Surg. 34, 1056-1057 (2008).

[33] Z. Zalevsky "Extended depth of focus imaging: a review," SPIE Reviews 1, 018001-1-018001-11 (2010). 